\title{
Novel "inverse sensitivity" enzyme-linked crystal-growth assay to detect ultralow PSA levels
}

Amidst the storm gathering around the use of PSA screening to detect early prostate cancer, a team from Imperial College London has developed a novel assay that is able to measure serum PSA at concentrations down to $10^{-18} \mathrm{~g} / \mathrm{ml}$, and that could be a useful tool in detecting biochemical recurrence of the disease at an early stage.

The team developed a novel enzymecontrolled crystal-growth system, wherein the formation of silver crystals on a gold nanosensor was affected by the activity of the glucose oxidase (GOx) enzyme. In this system, low concentrations of GOx result in slow growth of silver crystals, which favours the formation of a homogenous coating of silver on the gold nanosensors. However, high concentrations of the enzyme cause nucleation of the silver crystals and the formation of free-standing nanocrystals. The different rates of crystallisation can be measured by a shift in the localized surface plasmon resonance (LSPR) of the gold nanosensors, measurable using colorimetry. This system can then be used as an assay by linking it to antibodies for a specific analyte and measuring the

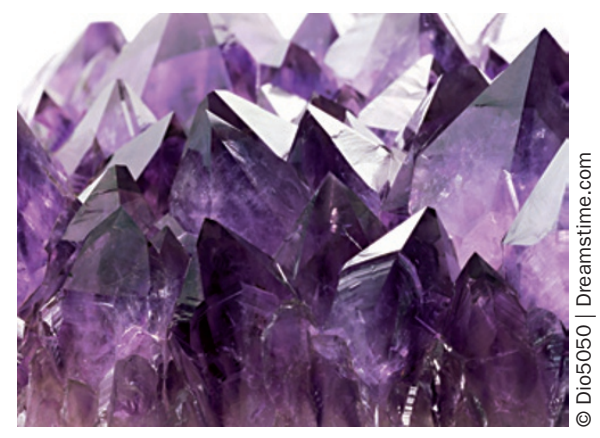

LSPR, which is inversely proportional to the amount of analyte detected in the sample - that is, the lowest concentrations of the target produce the highest signal, but an absence of analyte produces no signal at all.

PSA was chosen as the perfect biomarker to test the system, as ultralow serum PSA levels are a clinically useful marker of biochemical recurrence of prostate cancer, but are currently difficult to detect.

"Conventional approaches such as ELISA are not sensitive enough to detect PSA at this ultralow level," Molly Stevens, who led the study, told Nature Reviews Urology. "Even with other sophisticated ultrasensitive approaches, the presence of the biomarker at ultralow concentrations would de facto generate a tiny signal proportional to the concentration of the target protein, which might be easily masked by interferences found in body fluids". The team modified the gold nanosensors with polyclonal antibodies against PSA, which was then detected with secondary antibodies bound to GOx. The resulting assay could detect concentrations of PSA as minute as $1 \mathrm{ag} / \mathrm{ml}$-one order of magnitude lower than proposed digital ultrasensitive assays.

The authors believe that the discovery of this inverse sensitivity phenomenon represents a breakthrough in diagnostic sensors, enabling the detection of biomarkers at ultralow levels with a high degree of confidence. They now plan to use samples from patients with prostate cancer to assess the practicality of using inverse sensitivity to diagnose biochemical recurrence of the disease.

\section{Annette Fenner}

Original article Rodríguez-Lorenzo, L. et al. Plasmonic nanosensors with inverse sensitivity by means of enzymeguided crystal growth. Nat. Mater. doi:10.1038/nmat3337 